\title{
A Power-based Method for Improving the ODMRP Protocol Performance in Mobile Ad-hoc Networks
}

\author{
${ }^{1}$ Arash.Ghafouri, ${ }^{2}$ Ahmad Ghasemi, ${ }^{3}$ Mohammad Reza. Hasani Ahangar \\ ${ }^{1}$ Imam Hossein University/ Department of Computer Engineering,Tehran, Iran \\ ${ }^{2}$ Imam Hossein University/ Department of Computer Engineering \\ ${ }^{3}$ Imam Hossein University/ Department of Computer Engineering, Tehran, Iran
}

Received: 13 October 2017; Accepted: 17 January 2018; Published: 08 March 2018

\begin{abstract}
Mobile AD hoc Networks as a special type of wireless networks have received special attention due to having special features such as no need for central management, no need for infrastructure and high mobility capability and can be used in cases where creating an effective communication infrastructure is not cost-effective or is practically impossible, such as conferences, such as battles and communications after natural disasters. Several routing protocols are proposed for these networks. ODMRP protocol is one of the most famous and used protocols in Mobile AD hoc networks. This study was carried out aimed to discuss this routing protocol and then provide a new routing method for this protocol for increasing its efficiency. In the ODMRP protocol, the optimal route is selected based on the shortest route. In wireless communications and getting the nodes away from each other, the received signal levels are weakened and may result in loss of data, and in practice, the shortest path that works based on the number of hops loses its effectiveness. In the proposed protocol, the route is selected based on the received signal strength level. According to the simulation results, the performance of the proposed protocol increases by decreasing control overhead and increasing the packet delivery rate compared to the original protocol.
\end{abstract}

Index Terms: Wireless networks, Mobile AD hoc Networks, Routing protocols, ODMRP protocol

(C) 2018 Published by MECS Publisher. Selection and/or peer review under responsibility of the Research Association of Modern Education and Computer Science

\section{Introduction}

Today, wireless networks have received much attention and are widely used due to their vast applications and

* Corresponding author.

E-mail address: krghafouri@ihu.ac.ir, ghasemi@toluenet.ir, mrhasani@ihu.ac.ir 
ease of use and these networks are constantly growing. Simultaneously, with the use of these networks, the services and quality of this network should also be improved day by day and. In terms of architecture, wireless networks can be divided into two categories, including infrastructure-based networks and infrastructure-less networks. Infrastructure-less networks or Mobile AD hoc networks only include mobile nodes with no central stations, wired connections or network infrastructures [1]. In the Mobile AD hoc networks, nodes can move freely on the network. In these types of networks, nodes in the network are used as router in order to send information. One of the main features of Mobile AD hoc networks is continuous change in the configuration of these networks due to the mobility of the nodes. Also, restrictions on network resources such as bandwidth and power supply can be noted as other features of these networks. The issue of discovering and maintaining the route is of particular importance due to the unstable nature of Mobile AD hoc networks, [2].

We are trying to find an affordable routing method due to the two factors of lack of high bandwidth and limited energy in each node. The packets in this network are transferred during a series of continuous storage and retransmission operations by a set of intermediate and middle nodes. The main goal of the routing is to transfer a data packet from the source to the destination in a secure way [3]. In addition, another goal of the routing is to minimize latency. Several routing algorithms are presented these networks, each of which has its own disadvantages, advantages and characteristics. Routing protocols are divided into two types in terms of planning method:

\section{Table Driven Protocols}

In this routing method, each nodes uses routing information by storing the local information of other nodes in the network and this information is used to transmit data through different nodes. This protocol includes DSDV, WRP, CSGR and STAR methods.

\section{On Demand Protocols}

In this method, routing between nodes is created only when is needed for packet routing. This protocol includes SSR, DSR, TORA, AODV and RDMAR methods. Also, a third type can be considered called Hybrid Protocol. This type combines the two methods above. In these protocols, vector-distance routing method is used to find the shortest route, and it reports routing information only when there is a change in the network topology. The protocols include the following methods SMR, AOMDV, AODVM, ZD-AOMDV, and IZM-DSR. One of the most popular routing algorithms for Mobile AD hoc Networks is the ODMRP algorithm. The ODMRP protocol is designed by the WAM Laboratory at UCLA University [4].

\section{Related Works}

Given that the odmrp protocol is one of the most widely used protocols, there are many ways to improve the performance of the odmrp protocol. In most of these cases, we have been working to reduce the routing weaknesses of this protocol. By[5] limiting the flooding of the packet Route request reduces the control overhead of messages and thus reduces latency to the end .

Authors in [6] present A way to reduce control overhead in ODMRP .The Enhanced ODMRP (EODMRP) proposed a dynamic refresh interval for the multicast mesh based on the network feedback on real disconnections experienced by the multicast network members. Veritably, EODMRP decreased the network control overhead at the cost of obtaining a low packet delivery ratio especially in high mobility conditions of the network.

In [7] researchers presented a newm ethod. this method based on path stability is proposed, which uses the mechanism of Multipoint Relay (MPR) to limit flooding, and puts forward neighbor change ratio to choose three comparatively stable routes.As the result of the improvement, this mechanism optimizes overhead. 
In another article [8] Changes in routing criteria routing such as packet size, group size, action range and mobility of nodes has been studied. ODMRP uses single path to forwarding packets and this cause to Packet Delivery Ratio reduction in destination. The proposed method in this article uses multi coded paths technique. Thus due to route diversity and overcoming single route breakage, that can improve Packet Delivery Ratio from source to destination.

In another research [9] an enhancement to ODMRP is proposed referred to as fuzzy logic based Rate Control ODMRP (FRC-ODMRP). FRC-ODMRP attempts to adapt the arrival rate from upper layers to the state in the network by using feedback information from receivers of the multicast group. Accordingly, source comes up with a decision whether to increase or decrease its transmission rate based on information collected from the receivers. In this research, delay and packet delivery ratio reconsidered as indicators of congestion in addition to number of received packets.

\section{a. Introduction of ODMRP protocol}

On-Demand Multicast Routing Protocol is a multicast protocol and operates in an On-Demand manner [10]. This protocol uses the concept of a forwarding group to build a mesh for each multicast group [11]:

The most important features of this protocol include: simplicity, high strength in the case of disconnection error and movement of nodes due to the mesh structure, no need to send no explicit control packet when the nodes join the group or leave the network, low memory overhead, use of new route for maintenance and exploitation of multiple and duplicate routes, the ability to increase the number of network nodes, the lack of dependence on single cast routing protocols. The disadvantages of this protocol include: Repetitive send of data due to mesh configuration, dependency of route repair to Join Query [12] that are sometimes propagated by the source. ODMRP is a mesh-based multicast protocol in which MANETs is used [13]. This protocol creates a group to transfer the node considering the shortest routes among members of the group [14]. Then it uses the created group to transfer data between members of the group. The nodes in the ODMRP can freely join the group without any information message or leave the group [15]. When a source sends the flooding of Join Queries, any node that receives a non-duplicate Join Query stores the upstream node's address and retransmits the packet. This will continue until a route is discovered from origin to destination. If a multicast destination node receives a Join Query, it initially creates a Join Reply and then propagates it. Each node that receives this Join Reply examines whether it is placed on the route to source. If the answer is yes, it will re propagate the package again, and will continue until the packet is received by the source. The ODMRP protocol creates and updates the routes by using the flooding propagation of the Join Queries. However, excessive flooding of limited bandwidth destroys Mobile AD hoc Networks and creates a control overhead. In addition, the flooding often causes congestion and collision. So finding the optimal flooding interval is critical in ODMRP performance. In the above cases, the ODMRP protocol maintains members of the multicast group using a soft approach. Any specific management messages aren't needed to join or leave the group, and nodes can apply to leave the group without any prior announcement. However, this method in the ODMRP protocol has the following problems and disadvantages, some of the most important are:

1. This method does not allow reproduction of the Join Query (JQ) for back in the learning process, so it will be difficult to find the optimal route back.

2. This method does not have a control mechanism for the physical layer, so the nodes send with constant power and constant propagation rates.

3. There is no mechanism to detect a node with better power for sending, that is, the shortest path is only component necessary to select the next node for routing.

4. Getting the nodes away from each other, their power received decreases and the potential for loss of packet information is increased. This leads to resend the packets due to congestion and congestion.

5. The ODMRP protocol only works based on the lowest hop [16] and this will sometimes be associated with problems. 
Figure 1 shows the overall performance of the ODMRP protocol to send Join Query.

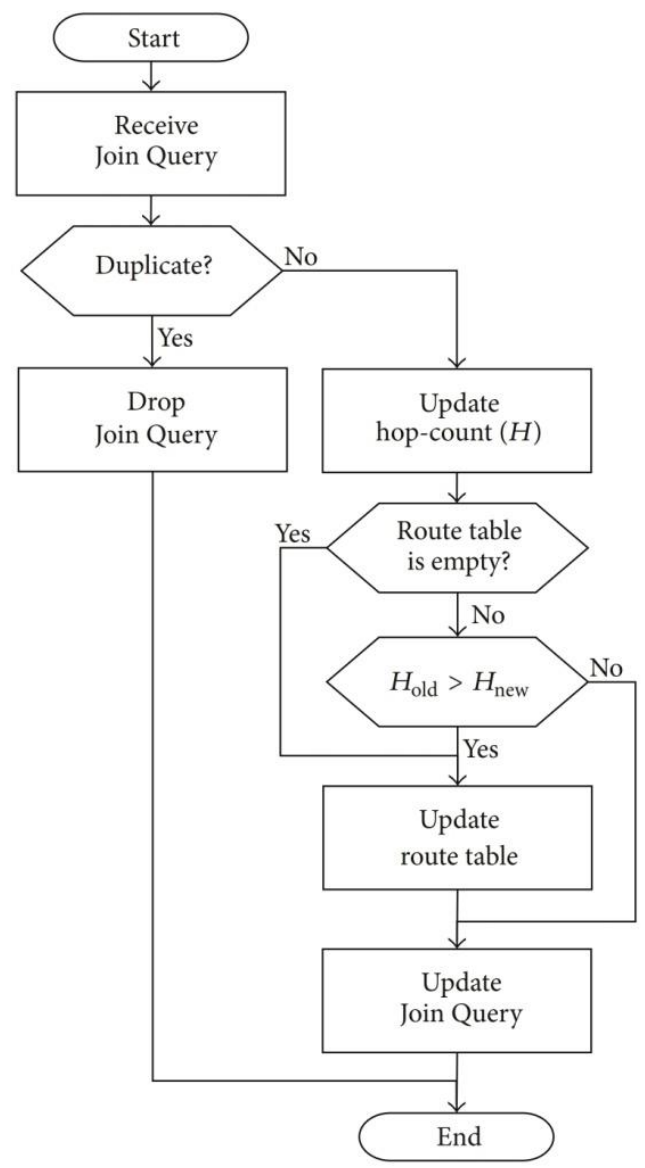

Fig.1.The function of the ODMRP protocol in sending a Join Query

\section{Proposed method}

In this section, we explain our proposed method in order to provide a method for improving ODMRP routing performance. In the proposed routing protocol, the parameters of power and signal strength of the received signal are also considered in addition to mentioned ones. The new protocol with this feature is known as Power Awareness ODMRP (PA-ODMRP).

The proposed method will lead to reduce the computational overhead and, as a result, increase packet delivery ratio. In addition, the use of this method will reduce the loss of packets, because in wireless communication and routing, getting the nodes away from each other, the power level of the received radio signal has dropped, and the less or non-acceptable power level reaches to the recipient's node. This will lead to loss the packets, and the sender's node will have to re-send the packets, so it leads to traffic, bandwidth consumption, and create a queue in the network. If with higher power levels are used in routing, the problem above can be solved. The proposed PA-ODMRP method, unlike the ODMRP protocol, is not based on the shortest route, and the best route in this protocol will have a higher power. The routing mechanism of this method will be discussed in the next section. 


\section{a. PA-ODMRP protocol routing mechanism}

Since the only criteria for choosing the best route is the shortest path (the route with has less hop) in the protocols that work based on the distance vector, other factors may not be considered and it may be associated with problems. In the proposed method which is related to the wireless and Mobile AD hoc networks, the main focus is on the power level transmitted and received by each node. The problem arisen with choosing the shortest route as the best route can be influenced by the presence of nodes with a long distance of each other, which lead to failure to reach the desired power level to the receiver node. In the next section, we describe this issue and relevant challenges. As shown in Fig. 2, there are two routes from the source node $S$ to the destination D.

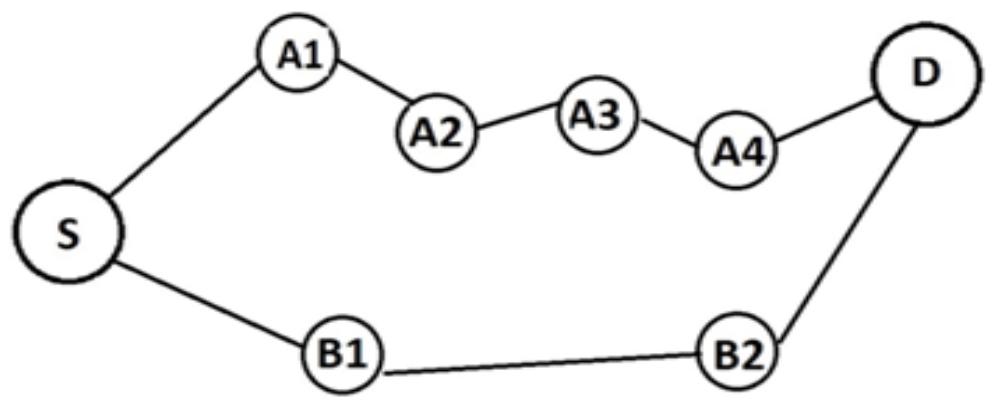

Fig.2. Comparison of routes and power received

The first route by nodes A1, A2, A3, and A4, and the second route by nodes B1 and B2 Obviously, in On-Demand Multicast Routing protocol, Route B is selected as the shortest and optimal route for sending, because the route has a less hops. But in practice because the distance between B1 and B2 or B2 to D may be high, it is likely that the acceptable signal level doesn't reach to the receiver node and will loss the information packets during sending. This will cause the sender has to retransmit the packets, which will result in creating a queue and delay in the delivery of packets. If in the route A, despite the higher number of hops, due to the closeness of the nodes, each node receive a power at a high level, and the loss of information due to the low level of the signal won't be possible. Now, if high-power routes in both sending and receiving modes are in the top priority, the routing criterion is changed from the less number of hops to the high-power routes, we can say that the problem of losing information packets due to low power will be minimized. The PA-ODMRP routing mechanism is as follows:

1. First, along with the Join Query to find the route, the power level received is also sent to the nodes, meaning each node, along with the Join Query, sends the power level received to the neighboring nodes. In accordance with the ODMRP protocol, all nodes which receive this packet, if they are not duplicate ones, and have not expired will publicly propagate this message. The subject arisen in ODMRP is that in this protocol, routing and Query for the creation of a route to the destination is done based on Query, but when a route is established to a destination, this route is periodically updated, and this is done by sending Join Query in the flooding manner periodically. In the proposed PA-ODMRP protocol, the route is updated periodically and according to the original ODMRP protocol.

2. If there is no duplicate query, and the time has not expired, the node will record this query and update its routing table and recall the return route to node sender. The additional work done in the new PA-ODMRP protocol is that in addition to the above, the receiver node sends its power level received to 
the next node. A node of neighboring nodes that receives the route Query packet checks it, and, if it is eligible (not duplicate and does not end time), in addition to the items in the main protocol, stores the power level in a table.

3. The publicity propagation process of the Route Query and the power level are sent repeatedly to identify the existing routes to the destination.

4. Given that every node stores the amount of power received from the sender's node and sends it to the next node, these powers are sent from the previous nodes for the next node. Figure 3, shows that the first node only sends its received power to the second node. But the second node, in addition to its power received, information related to the power received by the first node sends to the third node. Similarly, the third node sends the information received by itself, second and first nodes, as a table to the fourth node. This process will continue in this way, and eventually information received by all nodes will reach to the destination node.

5. At the end and after the query packet arrives at the destination, a route will be selected as the optimal route which has the highest average amount of power received. The average power received is obtained by calculating the sum of power received by all recipients to the destination divided by their number.

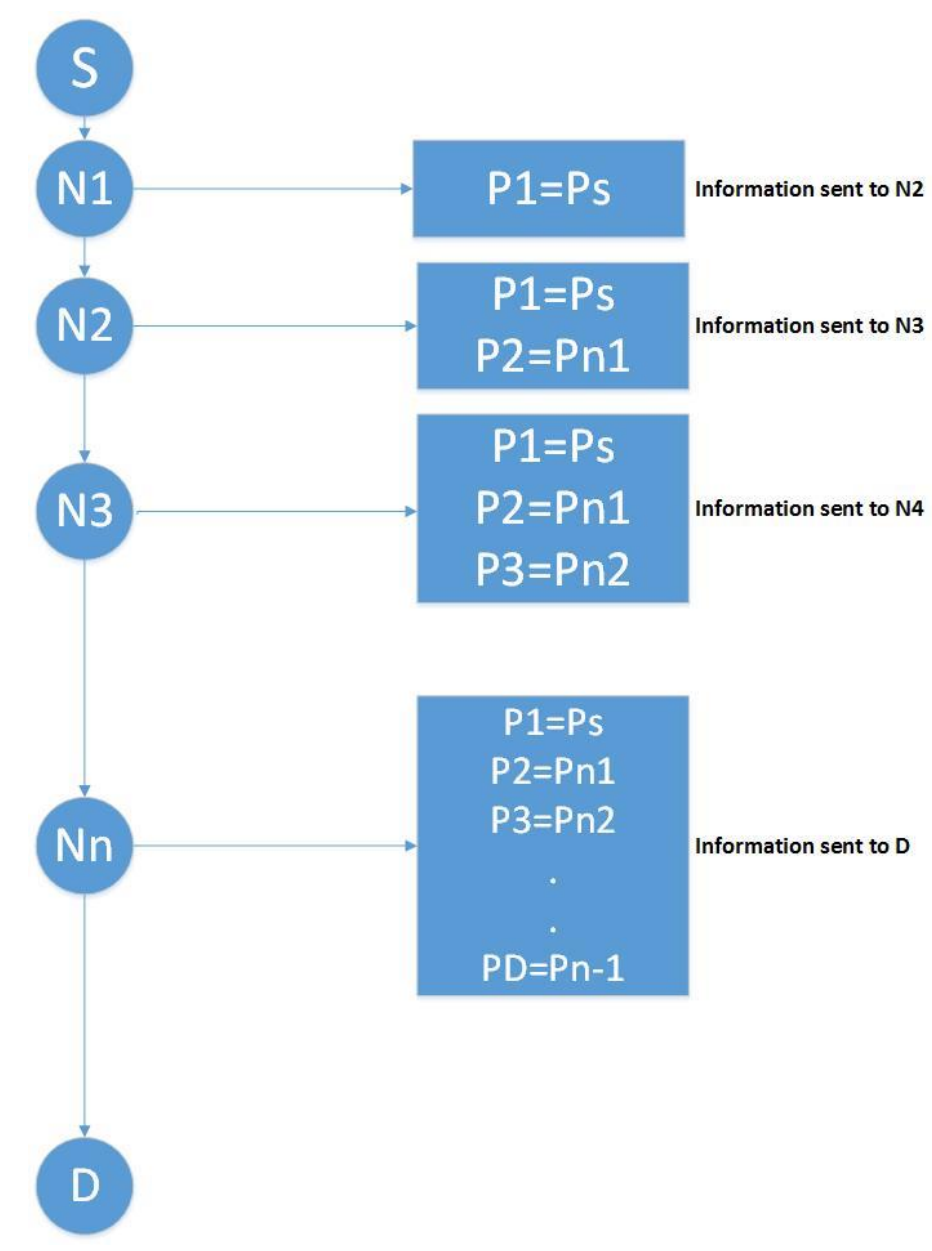

Fig.3. Recording the power received by each node 


\section{Simulation and comparing the proposed protocol}

The results of simulation and comparison of two PA-ODMRP and ODMRP protocols are in three fields are discussed in this section. Comparison of control overhead changes with increasing data rates (data packets per second), comparison of control overhead changes with increasing number of nodes, comparison the percentage of packet delivery with increasing speed in this simulation is studied. The above has a significant impact in speed and efficiency of the network, and packet delivery rate will increase dramatically by reducing control overhead and an increase in the packet delivery rate.

\section{a Comparison of control overhead with data rate changes}

Here, the control overflow is defined as the total number of control packets on the total number of delivery packages. Therefore, whatever the control overhead is lower, then the lower control information will be exchanged in the messages transfer process. This is shown in Figure 4.

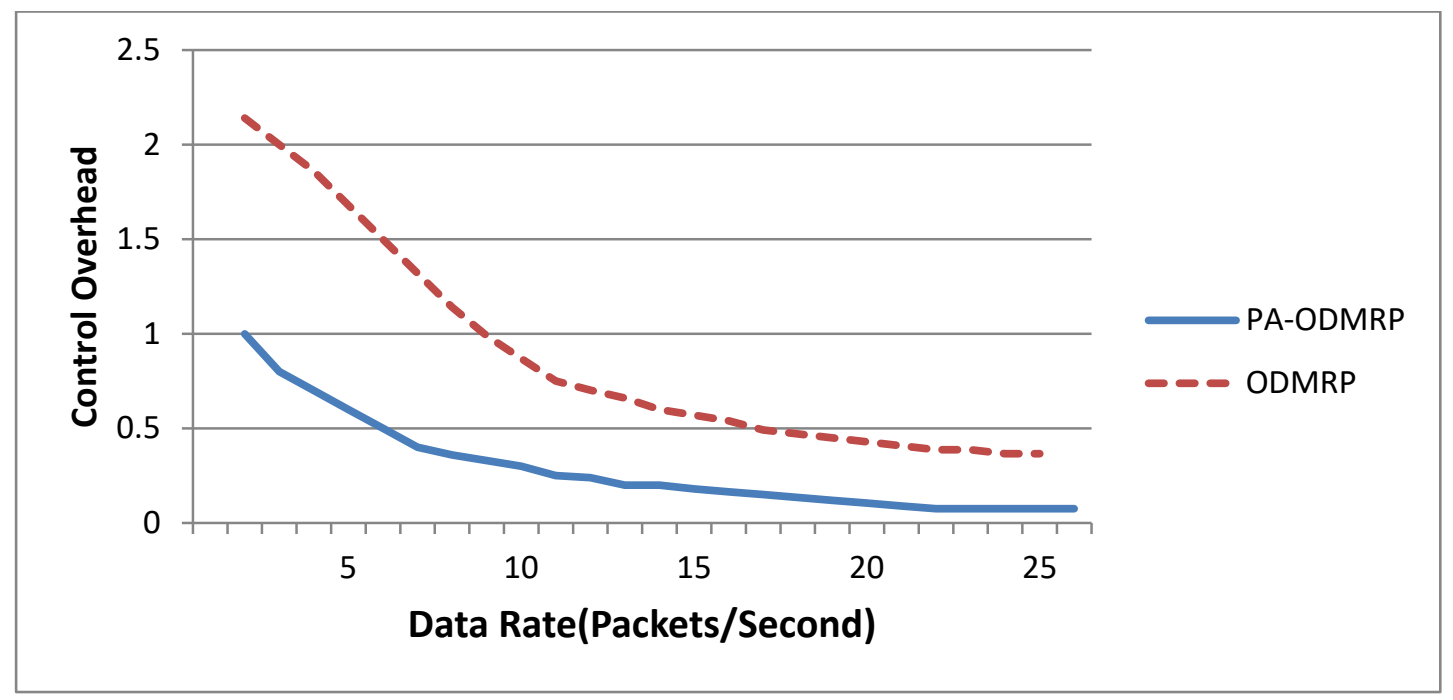

Fig.4. Data Rate (control packets per second)

As it can be observed in the figure, control overhead reduces by increasing the data rate in both control protocols. The PA-ODMRP proposed protocol has better results compared with the original ODMRP protocol. The control overhead at a low data rate is about $100 \%$ and at a high data rate has reduced about $40 \%$ compared with the original protocol.

\section{$b \quad$ Comparison of control overhead with changes in number of node}

Always, one of the concerns has been how to react to the increased number of nodes by the protocol. In the ODMRP protocol, given the formation of forwarding groups as well as the mesh-based behavior of the protocol, if the number of nodes increases, control overhead will reduce. But this is more observed in the proposed PA-ODMRP protocol, and given the lower loss of information packets, the protocol has fewer control overhead compared with the original ODMRP protocol. This is shown in Figure 5. The control overhead in the proposed protocol, on average, has reduced by 30 percent compared with the original protocol. 


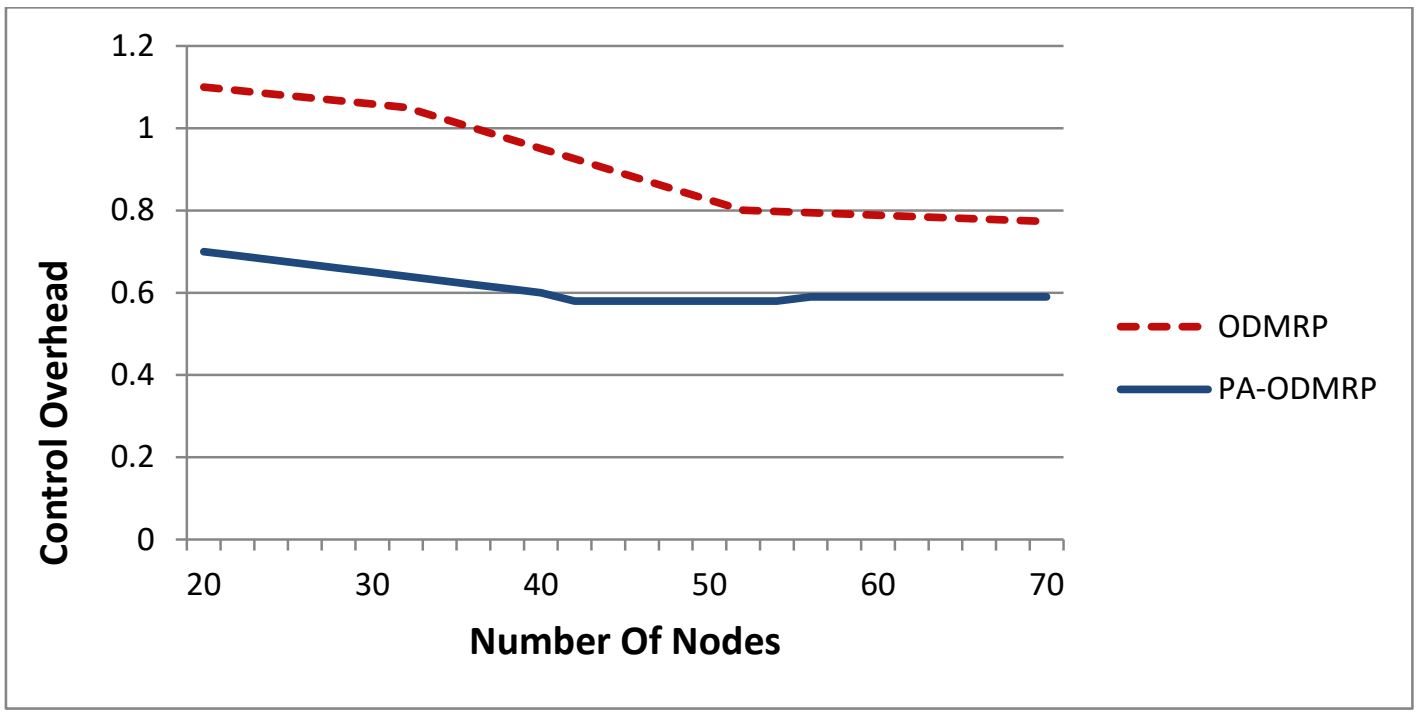

Fig.5. Comparison of control overhead with changes in the number of nodes

\section{c Comparing the percentage of packet delivery with changes in the nodes speed}

In vehicles, no change must be made in the package delivery rate by increasing the speed of a node. As it can be observed in Figure 6, in both protocols, packet delivery rate decreases with increasing speed, but firstly this decrease is not important and, secondly, the decrease rate is very low. Figure 6 shows that the packet delivery rate in the proposed protocol at higher speeds is higher at least $10 \%$ than the original protocol.

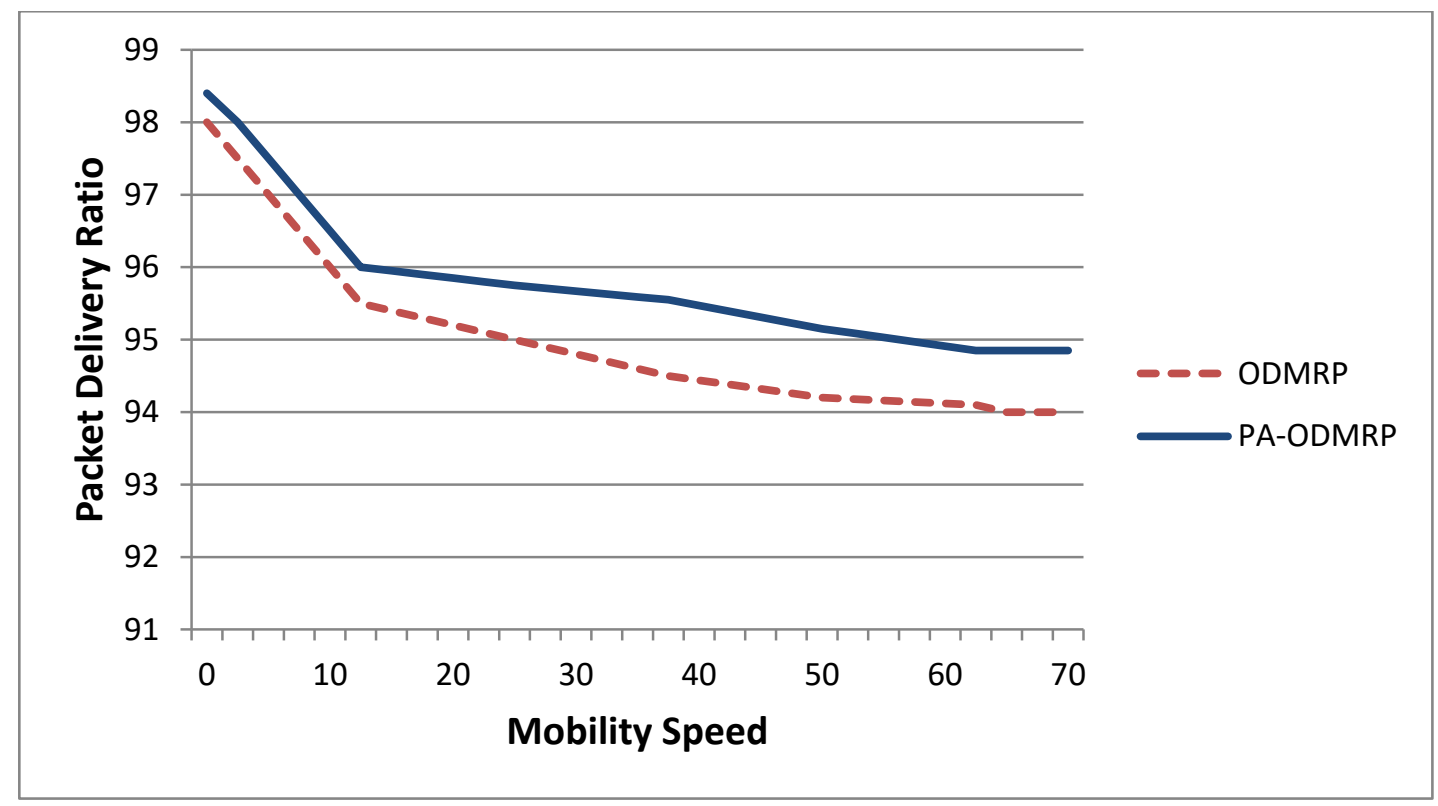

Fig.6. Comparison of packet delivery rates with node speed changes 


\section{Conclusions}

The ODMRP protocol has disadvantages in spite of its positive features such as simplicity, high strength in the case of disconnection error and movement of nodes, no need to send no explicit control packet when the nodes join the group or leave the network, having single-cast and multicast capabilities. One of these disadvantages is that routing is done based on the shortest route, regardless of the possibility of information packets loss due to the weakening of the strength of the radio signal. Routing is done using the power component received from each node in both the proposed method and the PA-ODMRP protocol. In this method, by specifying the power received from each node, a route with higher power level in the receiver will be used for dealing with information packets loss. According to simulation results, the PA-ODMRP protocol has a better performance than the main protocol in terms of reducing the computational overhead by $30 \%$ and increasing the percentage of delivery packages rate by $10 \%$.

\section{References}

[1] C. S. R. Murthy and B.S. Manoj, "Ad Hoc Wireless Networks Architectures and Protocols", PRENTICE HALL, 2004.

[2] S. Yang, J. Wu, "New technologies of multicasting in MANET," Design and Analysis of Wireless Networks, Y. Xiao and Y. Pan, Eds,. Nova Science Publishers, Baltimore, MD, USA, 2005.

[3] Ma Weihua, Research of multicast routing protocol in Ad Hoc network[D].Beijing: master degree thesis of The national defense science and technology university, 2009.

[4] L.M. Feeney, "A taxonomy for Routing Protocols in Mobile Ad-hoc Network". www.sics.se/ lmFeeney/huc.pdf, 2001.

[5] Mahdi KHazai, Reduced control overhead and load balancing in the odmrp multiprocessor routing protocol,Third National Conference on Computer Engineering and Information Technology, Hamedan, Iran, 89

[6] Darabkh and Ola A. Alsukour , Novel Protocols for Improving the Performance of ODMRP and EODMRP over Mobile Ad Hoc Networks Hindawi Publishing Corporation International Journal of Distributed Sensor Networks, 2015.

[7] Yang Yanga, Cao Jieb, Improvement on Multicast Routing Protocol ODMRP Based on Path Stability, International Conference on Computer Science and Information Technology, 2011.

[8] Shapour Joudi Begdillo, Mehdi Asadi, Abolfazl Toroghi Haghighat, Improving Packet Delivery Ratio in ODMRP with Route Diversity, IJCSNS International Journal of Computer Science and Network Security, 2007.

[9] Alireza Shams Shafigh, Beatriz Lorenzo, Savo Glisic, Fuzzy Logic Based Rate Control Scheme for ODMRP in Mobile Ad hoc Networks Online, Automatika Journal for Control, Measurement, Electronics, Computing and Communications, 2014.

[10] S. J. Lee, M. Gerla, and C. C. Chiang, "On-Demand Multicast Routing Protocol” Proceeding of IEEE WCNC, 1999.

[11] Abdollahi, K., A. Shams Shafigh and A.J. Kassler, Improving performance of ODMRP by Deleting Lost Join Query Packets. ACIT, Spain, 2010.

[12] Pathirana, D., Resilient On Demand Multicast Routing Protocol. Master of Science thesis, ROCHESTER Institute of Technology, 2007

[13] Esmaeili T, Lari M, Akbari Jahani E, Mohamadi S, "Proposing an approach for routing overhead reduction in mobile ad hoc networks," Electronic Computer Technology, International Conference on , May, 2010.

[14] Y. Yi, S.-J. Lee, W. Su, M. Gerla, Mobile ad hoc networking (manet): Routing protocol performance issues and evaluation considerations, Internet-Draft (http://tools.ietf.org/id/draft-ietf-manet-odmrp-04.txt), 
2002.

[15] C. Chiang, M. Gerla, L. Zhang, Forwarding group multicast protocol (fgmp) for multihop, mobile wireless networks, 1998.

[16] S.-J. Lee, W. Su, M. Gerla, Wireless ad hoc multicast routing with mobility prediction, Mob. Netw., 2001.

\section{Authors' Profiles}

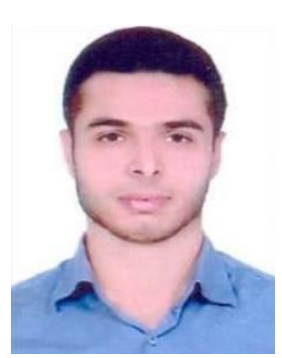

Arash Ghafouri is a researcher of computer engineering in the school of computer engineering of Imam Hossein University. He was born in Tehran, Iran in 1990. He received his Master of Science in computer engineering with area of study of distributed systems from Iran University of Science and Technology in 2014. His current research interests include distributed and high performance computing, virtualization technology, network security, and data science.

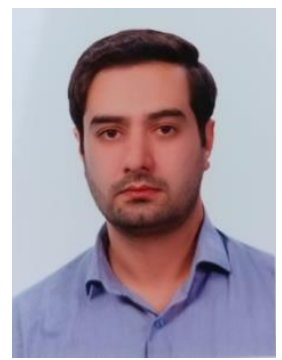

Ahmad Ghasemi received his B.S. degree in Electronic engineering from Mohaddes Noori University,Noor,Iran, in 2006, and now is student in M.S in IT engineering in Imam Hoseyn University, Tehran, Iran. His research interests cover Mobile Ad hoc Network (MANET) and specially Power in this Network.

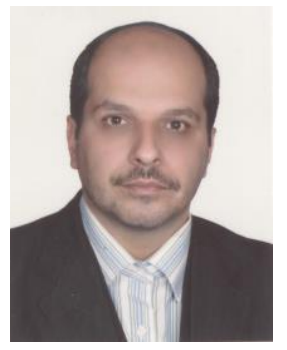

Mohammad Reza Hasani Ahangar is an associate professor of computer engineering in the school of computer engineering of Imam Hossein University. He directs the research laboratory of artificial intelligence. He is mainly interested in intelligent systems, solutions, and applications, particularly for use in various fields of science. He received his $\mathrm{PhD}$ in computer engineering from Iran University of Science and Technology in 2011.

How to cite this paper: Arash.Ghafouri, Ahmad Ghasemi, Mohammad Reza. Hasani Ahangar," A Power-based Method for Improving the ODMRP Protocol Performance in Mobile Ad-hoc Networks", International Journal of Wireless and Microwave Technologies(IJWMT), Vol.8, No.2, pp. 27-36, 2018.DOI: 10.5815/ijwmt.2018.02.03 